\title{
A family of supermodular Nash mechanisms implementing Lindahl allocations ${ }^{\star}$
}

\author{
Yan Chen \\ Department of Economics, University of Michigan, Ann Arbor, MI 48109-1220, USA \\ (e-mail: yanchen@umich.edu)
}

Received: April 27, 2000; revised version: January 16, 2001

Summary. We present a family of mechanisms which implement Lindahl allocations in Nash equilibrium. With quasilinear utility functions this family of mechanisms are supermodular games, which implies that they converge to Nash equilibrium under a wide class of learning dynamics.

Keywords and Phrases: Public goods mechanisms, Supermodular games.

JEL Classification Numbers: H41, C62, D83.

\section{Introduction}

The presence of public goods seriously challenges traditional or "natural" solutions for the allocation of private goods. Important policy questions, of whether we can rely on the market to provide optimal amounts of public goods such as air pollution, and how much we can rely on "natural" processes such as voluntary contribution to solve environmental problems, boil down to fundamental issues about human nature, i.e., about whether people are selfish or cooperative. The vast experimental literature on voluntary provision of public goods shows that

\footnotetext{
* I thank John Ledyard, David Roth and Tatsuyoshi Saijo for discussions that lead to this project; Klaus Abbink, Beth Allen, Rachel Croson, Roger Gordon, Elisabeth Hoffman, Wolfgang Lorenzon, Laura Razzolini, Sara Solnick, Tayfun Sönmez, William Thomson, Lise Vesterlund, seminar participants in Bonn (EDP), Hamburg, Michigan, Minnesota, Pittsburgh, Purdue and participants of the 1997 North America Econometric Society Summer Meetings (Pasadena, CA), the 1997 Economic Science Association meetings (Tucson, AZ), the 1998 Midwest Economic Theory meetings (Ann Arbor, MI) and the 1999 NBER Decentralization Conference (New York, NY) for their comments and suggestions. Special thanks go to Matthew Jackson and an anonymous referee for pointing out an error in the proof of Theorem 2 in a previous version. The hospitality of the Wirtschaftspolitische Abteilung at the University of Bonn, the research support provided by Deutsche Forschungsgemeinschaft through SFB303 at the University of Bonn and NSF grant SBR-9805586 are gratefully acknowledged.
} 
"although inexperienced subjects can be led to provide large contributions in one-time decisions with the use of relevant discussions, one cannot rely on these approaches as a permanent organizing feature without expecting an eventual decline to self-interested behavior. ... Since 90 percent of subjects seem to be responsive to private incentives, it is possible to create new mechanisms which focus that self-interest toward the group interest." (p. 173, "Public Goods: A Survey of Experimental Research" in The Handbook of Experimental Economics, 1995.)

These "new mechanisms", i.e., incentive-compatible mechanisms for public goods provision, are innovative allocation-taxation rules which can achieve a Pareto-optimal allocation of resources with public goods. It is well known that it is impossible to design a mechanism for making collective allocation decisions, which is informationally decentralized (i.e., mechanisms which only use the agents' own messages), non-manipulable (or dominant strategy incentivecompatible), and Pareto optimal ${ }^{1}$. There are many mechanisms which preserve Pareto optimality at the cost of non-manipulability, some of which preserve "some degree" of non-manipulability. In particular, some mechanisms have been discovered which have the property that Nash equilibria are Pareto optimal ${ }^{2}$. These can be found in the work of Groves and Ledyard (1977), Hurwicz (1979), Walker (1981), Tian (1989), Kim (1993), Peleg (1996) and Falkinger (1996).

Nash implementation theory has mainly focused on establishing static properties of the equilibria. When a mechanism is implemented among real people, i.e., boundedly rational agents, however, we expect the actual implementation to be a dynamic process, starting somewhere off the equilibrium path. Following Hurwicz (1972), one could interpret the Nash equilibrium strategies of a game form as the stationary messages of some decentralized learning process. The fundamental question concerning implementation of a specific mechanism is whether the dynamic processes will actually converge to one of the equilibria promised by theory.

The few theoretical papers on the dynamic properties of public goods mechanisms have been using very specific learning dynamics to investigate the stability of mechanisms. Muench and Walker (1983) and de Trenqualye (1988) study the convergence of the Groves-Ledyard mechanism under Cournot best-reply dynamics. De Trenqualye (1989) and Vega-Redondo (1989) propose mechanisms for which the Cournot best-reply dynamics is globally convergent to the Lindahl equilibrium $^{3}$ outcome. Kim (1993) proposes a mechanism which implements Lindahl allocations and remains stable under the gradient adjustment process given quasilinear utility functions. One exception is Cabrales (1999) who studies

\footnotetext{
1 This impossibility has been demonstrated in the work of Green and Laffont (1977), Hurwicz (1975), Roberts (1979) and Walker (1980) in the context of resource allocation with public goods.

2 Other implementation concepts include perfect Nash equilibrium (Bagnoli and Lipman, 1989), undominated Nash equilibrium (Jackson and Moulin, 1991), etc.

3 A Lindahl equilibrium for the public goods economy is characterized by a set of personalized prices and an allocation such that utility and profit maximization and feasibility conditions are satisfied.
} 
dynamic convergence and stability of the canonical mechanism in Nash implementation and the Abreu-Matsushima mechanism (1992) under "naive adaptive dynamics"4.

Recent experimental studies on learning strongly reject the Cournot best-reply learning model in favor of other models (e.g., Boylan and El-Gamal, 1993). So far there has been no experimental investigation of the gradient adjustment process, even though it has been used fairly extensively in the theoretical research on stability of games (Arrow and Hurwicz, 1977). Experimental research on learning is still far from reaching a conclusion with regard to a single "true" learning model that describes all adaptive behaviors. Furthermore, there is strong evidence that individual players adopt different learning rules under different circumstances (El-Gamal and Grether, 1995). It is therefore desirable to identify mechanisms which converge under a wide class of learning dynamics. This paper does so by focusing on mechanisms which are supermodular games.

The class of supermodular games ${ }^{5}$ has been identified as having very robust dynamic stability properties (Milgrom and Roberts, 1990): it converges to the set of Nash equilibria that bound the serially undominated set under a wide class of interesting learning dynamics, including Bayesian learning, fictitious play, adaptive learning, Cournot best response and many others. Therefore, instead of using a specific learning dynamic, we investigate whether we can find Nashefficient public goods mechanisms which are supermodular games.

The idea of using supermodularity as a robust stability criterion for Nashefficient mechanisms is not only based on its good theoretical properties, but also on strong experimental evidence. Chen (forthcoming) examines all experiments on incentive-compatible mechanisms for public goods. She finds that every experiment which converge to the Nash equilibrium prediction is a supermodular game, while none of the experiments which does not converge is a supermodular game. She proves that among the Nash-efficient public goods mechanisms the Groves-Ledyard mechanism is a supermodular game in quasilinear environments when the punishment parameter is above a certain threshold, while none of the Hurwicz (1979), Walker (1981) and Kim (1993) mechanisms is supermodular. These results are consistent with the experimental findings. The question remains whether we can find a Nash mechanism, which implements Lindahl allocation in a general environment and has a robust stability property (i.e., supermodular) in quasilinear environments.

In this paper, we propose a new family of mechanisms, which implement Lindahl allocations in Nash equilibrium in a general environment and are also supermodular games given quasilinear utility functions.

Section 2 introduces the environment. Section 3 discusses supermodular games. Section 4 introduces the new family of mechanisms and proves the implementation and stability results. Section 5 concludes the paper.

4 This is different from the adaptive learning in Milgrom and Roberts (1990). For a precise definition see Cabrales (1999).

5 See Section 3 for a formal definition. 


\section{A general public goods environment}

We will assume that there is one private $\operatorname{good} x$, one public good $y$, and $n \geq 3$ players, indexed by subscript $i$. Our results can be generalized to any number of public goods. Production technology for the public good exhibits constant returns to scale, i.e., the production function $f(\cdot)$ is given by $y=f(x)=x / b$ for some $b>0$. The assumption of constant returns to scale is made to simplify the production side of the story. Theorem 2 still holds under a general convex production function, however, the stability results (Proposition 1) might also depend on the parameters of the production function. Each player is characterized by a consumption set which is the nonnegative orthant in $\mathbb{R}^{2}, \mathscr{C}_{i}=\mathbb{R}_{+}^{2}$, a preference relation $\succeq_{i}$ on $\mathscr{C}_{i}$, and an initial endowment of the private good $\omega_{i}^{x} \in \mathbb{R}^{1}$. Let $E_{i}$ represent the set of individual preference orderings and initial endowments. We formally define $E_{i}$ as follows.

Definition 1 For each $i \in N$, let $E_{i}=\left\{\left(\succeq_{i}, \omega_{i}^{x}\right): \succeq_{i}\right.$ is transitive, complete, convex, continuous, and strictly increasing in private good $x$, and $\left.\omega_{i}^{x}>0.\right\}$

The $i^{\text {th }}$ agent's characteristics are determined by $e_{i} \in E_{i}$. The space of environments for the economy is $E=\Pi_{i=1}^{n} E_{i}$. An environment for the economy is represented by $e \in E$. The analysis for Nash implementation of the Lindahl allocations will be carried out in this general environment, for any $e \in E$. To prove the implementation result (Theorem 2) we need an additional assumption on the preference relation ${ }^{6}$.

Assumption 1 The upper contour sets are in the interior of the consumption space, i.e., for any $\left(x_{0}, y_{0}\right) \in \mathscr{C}_{i},\left\{(x, y) \in \mathscr{C}_{i} \mid(x, y) \succeq_{i}\left(x_{0}, y_{0}\right)\right\} \subset \mathscr{C}_{i}^{\circ}$.

Intuitively Assumption 2 requires that indifference curves do not hit the boundary of the consumption set. Many frequently used utility functions, such as the Cobb-Douglas utility functions, satisfy Assumption 2. For the dynamic stability analysis we will restrict the environment further to the class of quasilinear preferences. Note although the class of quasilinear preferences does not satisfy Assumption 2 it is straightforward to extend the implementation theorem to this class of environment.

Definition $2 E^{Q}=\left\{\left(\succeq_{i}, \omega_{i}^{x}\right) \in E: \succeq_{i}\right.$ is representable by a $C^{2}$ utility function of the form $v_{i}(y)+x_{i}$ such that $D v_{i}(y)>0$ and $-\infty<D^{2} v_{i}(y)<0$ for all $y>$ 0 , and $\left.\omega_{i}^{x}>0\right\}$, where $D^{k}$ is the $k^{\text {th }}$ order derivative.

An economic mechanism is defined as a non-cooperative game form played by the agents. The game is described in its normal form. In all mechanisms considered in this paper, the implementation concept used is Nash equilibrium. In the Nash implementation framework the agents are assumed to have complete information about the environment while the designer does not know anything about the environment.

\footnotetext{
${ }^{6}$ I thank William Thomson for suggesting this.
} 


\section{Supermodular games}

We first define supermodular games and review their stability properties. Then we discuss alternative stability criteria and their relationship with supermodularity.

Supermodular games are games in which each player's marginal utility of increasing her strategy rises with increases in her rival's strategies, so that (roughly) the player's strategies are "strategic complements". Supermodular games need an order structure on strategy spaces, a weak continuity requirement on payoffs, and complementarity between components of a player's own strategies, in addition to the above-mentioned strategic complementarity between players' strategies. Suppose each player $i$ 's strategy set $S_{i}$ is a subset of a finite-dimensional Euclidean space $\mathbb{R}^{k_{i}}$. Then $S \equiv \times_{i=1}^{n} S_{i}$ is a subset of $\mathbb{R}^{k}$, where $k=\sum_{i=1}^{n} k_{i}$.

Definition 3 A supermodular game is such that, for each player $i, S_{i}$ is a nonempty sublattice of $\mathbb{R}^{k_{i}}, u_{i}$ is upper semi-continuous in $s_{i}$ for fixed $s_{-i}$ and continuous in $s_{-i}$ for fixed $s_{i}, u_{i}$ has increasing differences in $\left(s_{i}, s_{-i}\right)$, and $u_{i}$ is supermodular in $s_{i}$.

Increasing differences says that an increase in the strategy of player $i$ 's rivals raises her marginal utility of playing a high strategy. The supermodularity assumption ensures complementarity among components of a player's own strategies. Note it is automatically satisfied when $S_{i}$ is one-dimensional. As the following theorem indicates supermodularity and increasing differences are easily characterized for smooth functions in $\mathbb{R}^{n}$.

Theorem 1 (Topkis, 1978) Let $u_{i}$ be twice continuously differentiable on $S_{i}$. Then $u_{i}$ has increasing differences in $\left(s_{i}, s_{j}\right)$ if and only if $\partial^{2} u_{i} / \partial s_{i h} \partial s_{j l} \geq 0$ for all $i \neq j$ and all $1 \leq h \leq k_{i}$ and all $1 \leq l \leq k_{j}$; and $u_{i}$ is supermodular in $s_{i}$ if and only if $\partial^{2} u_{i} / \partial s_{i h} \partial s_{i l} \geq 0$ for all $i$ and all $1 \leq h<l \leq k_{i}$;

Supermodular games are of interest particularly because of their very robust stability properties. Milgrom and Roberts (1990) proved that in these games the set of learning algorithms consistent with adaptive learning converge to the set bounded by the largest and the smallest Nash equilibrium strategy profiles. Intuitively a sequence is consistent with adaptive learning if players "eventually abandon strategies that perform consistently badly in the sense that there exists some other strategy that performs strictly and uniformly better against every combination of what the competitors have played in the not too distant past" ${ }^{\text {"7 }}$. This includes a wide class of interesting learning dynamics, such as Bayesian learning, fictitious play, adaptive learning, Cournot best-reply and many others.

Since experimental evidence suggests that individual players tend to adopt different learning rules (El-Gamal and Grether, 1995), instead of using a specific learning algorithm to study stability, one can use supermodularity as a robust stability criterion for games with a unique Nash equilibrium. For supermodular games with a unique Nash equilibrium, we expect any adaptive learning

\footnotetext{
${ }^{7}$ For a formal definition, see Milgrom and Roberts (1990).
} 
algorithm to converge to the unique Nash equilibrium, in particular, Cournot best-reply, fictitious play and adaptive learning. Compared with stability analysis using Cournot best-reply dynamics, supermodularity is much more robust and inclusive in the sense that it implies stability under Cournot best-reply and many other learning dynamics mentioned above.

There are two caveats for using supermodularity as a robust stability criterion. First, for supermodular games with multiple Nash equilibria, adaptive learning algorithms will converge to the set bounded by the largest and the smallest Nash equilibrium strategy profiles, however, players might not be able to learn to coordinate on a particular equilibrium. Van Huyck, Battalio and Beil (1990) examine a finitely repeated coordination game with seven Nash equilibria, which is supermodular. They found that with 14 to 16 players play tended to converge to the Pareto-dominated Nash equilibrium where each player chooses the minimum effort level. With two players, however, for 12 out of 14 pairs play converged to the Pareto-dominant Nash equilibrium where each player chooses the maximum effort level. Therefore, the equilibrium selection problem might depend on the group size and many other factors. Supermodularity does not help to predict which equilibrium will be selected.

Second, supermodularity is sufficient but not necessary for convergence. This implies that supermodular mechanisms with a unique Nash equilibrium ought to converge to the Nash equilibrium prediction fairly robustly, but mechanisms which are not supermodular could still converge to its equilibrium under some learning algorithms. In particular, supermodular games with a unique pure strategy Nash equilibrium is dominance solvable, but not vice versa. The robust convergence argument for supermodular games also applies to the larger class of dominance solvable games (Milgrom and Roberts, 1991). Dominance solvability is more inclusive but harder to check than supermodularity.

For a complete characterization of the dynamic stability of mechanisms, it is desirable to find both sufficient and necessary conditions for convergence under a wide range of learning dynamics. Since learning can differ from one context to another, we would need extensive experimental studies of human learning behavior under different mechanisms and the resulting repertoire of algorithms, calibrated against human responses, to cover various contexts. This is largely still an ongoing research enterprise. Once we have the accurately calibrated algorithms, we can restrict ourselves to the stability analysis based on these algorithms, and perhaps eventually characterize the sufficient and necessary conditions for these learning dynamics to converge.

\section{A new family of mechanisms}

Kim (1986) has shown that for any game form implementing Lindahl allocations there does not exist a decentralized adjustment process which ensures local stability of Nash equilibria in certain classes of environments. Chen (forthcoming) proves that none of the three existing game forms which implement Lindahl allocations in Nash equilibrium, Hurwicz (1979), Walker (1981) and Kim (1993), is 
supermodular in quasilinear environments. The question remains whether we can find a mechanism which implements the Lindahl allocations in Nash equilibrium in a general environment and also possesses a robust stability property at least with quasilinear preferences. In this section we provide a positive answer to this question by presenting a family of mechanisms which fulfill both roles.

In the mechanisms defined below the strategy space is two-dimensional. The new family of mechanisms, $\Gamma^{\gamma, \delta}$, is defined by two free parameters, $\gamma$ and $\delta$. Note when $\gamma=1$ and $\delta=0$ we obtain the Kim (1993) mechanism.

Definition 4 For mechanism $\Gamma^{\gamma, \delta}$, the strategy space of player $i$ is $S_{i} \subset \mathbb{R}^{2}$ with generic element $\left(m_{i}, z_{i}\right) \in S_{i}$. The outcome function of the public good and the net cost share of the public good for player $i$ are

$$
\begin{aligned}
& Y(m, z)=\sum_{k=1}^{n} m_{k}, \\
& T_{i}(m, z)=P_{i}(m, z) \cdot Y(m, z)+\frac{1}{2}\left(z_{i}-\sum_{k=1}^{n} m_{k}\right)^{2}+\frac{\delta}{2} \sum_{j \neq i}\left(z_{j}-\sum_{k=1}^{n} m_{k}\right)^{2}, \\
& \text { where } P_{i}(m, z)=\frac{b}{n}-\gamma \sum_{j \neq i} m_{j}+\frac{\gamma}{n} \sum_{j \neq i} z_{j}, \gamma>0 \text { and } \delta \geq 0 .
\end{aligned}
$$

The outcome function $Y(m, z)$ is the level of public good, and $T_{i}(m, z)$ is the cost share of player $i$ in terms of private good. A player's strategy $m_{i}$ is interpreted as the increment (or decrement) of the public good player $i$ would like to add to (or subtract from) the amounts proposed by others. Strategy $z_{i}$ is interpreted as player $i$ 's estimation for the public good level. If her estimation for the total level of public good is different from the sum of each individual's increment, she will be penalized by the quadratic difference, $\frac{1}{2}\left(z_{i}-\sum_{k=1}^{n} m_{k}\right)^{2}$; furthermore, she will be penalized by a constant multiple of the sum of the quadratic differences of other players, $\frac{\delta}{2} \sum_{j \neq i}\left(z_{j}-\sum_{k=1}^{n} m_{k}\right)^{2}$. Under the mechanism $\Gamma^{\gamma, \delta}$, since a player's choice of $\left(m_{i}, z_{i}\right)$ does not affect her price for the public good, $P_{i}(m, z)$, strictly increasing preferences in private good $x_{i}$ implies that a rational player will send her estimation of the sum of proposals, $z_{i}$, the same as the actual sum of proposals, that is, $z_{i}=\sum_{k=1}^{n} m_{k} \equiv y$, for all $i$. Therefore, in equilibrium the two quadratic punishment terms drop out.

Theorem 2 establishes that in a general environment the mechanism $\Gamma^{\gamma, \delta}$ implements the Lindahl allocations in Nash equilibrium.

Theorem 2 The mechanism $\Gamma^{\gamma, \delta}$ implements the Lindahl allocations in Nash equilibrium for any $e \in E$.

Proof. See Appendix.

Theorem 2 implies that in a general environment the Nash equilibrium of the mechanism $\Gamma^{\gamma, \delta}$ implements Lindahl allocations. Therefore, in equilibrium, the mechanism $\Gamma^{\gamma, \delta}$ is efficient, balanced and individually rational. A drawback 
of the mechanism is that off the equilibrium path, the mechanism might not be balanced. How serious this drawback is depends on how quickly the system converges when implemented, which is largely an empirical question.

Since Assumption 2 is needed to prove Theorem 2, it does not apply to the class of quasilinear preferences. However, it is straightforward to extend Theorem 2 to the class of quasilinear preferences.

Corollary 1 The mechanism $\Gamma^{\gamma, \delta}$ implements the Lindahl allocations in Nash equilibrium for any $e \in E^{Q}$.

Proof. See Appendix.

Corollary 2 The mechanism $\Gamma^{\gamma, \delta}$ has a unique Nash equilibrium for any $e \in E^{Q}$.

Proof. See Appendix.

If we restrict ourselves to the class of quasilinear preferences, the mechanism $\Gamma^{\gamma, \delta}$ has a particularly attractive stability property, i.e., within certain parameter ranges, it is a supermodular game. Therefore, it converges to the unique Nash equilibrium under a wide class of learning dynamics.

Proposition 1 The mechanism $\Gamma^{\gamma, \delta}$ is a supermodular game for any $e \in E^{Q}$ if and only if

$$
\delta \in\left[1-\min _{i \in N} \frac{\partial^{2} v_{i}}{\partial y^{2}},+\infty\right) \text { and } \gamma \in\left[1-\min _{i \in N} \frac{\partial^{2} v_{i}}{\partial y^{2}}+(n-1) \delta, n \delta\right] .
$$

Proof. (i) First, we prove that if $\delta \in\left[1-\min _{i \in N} \frac{\partial^{2} v_{i}}{\partial y^{2}},+\infty\right)$ and $\gamma \in[1-$ $\left.\min _{i \in N} \frac{\partial^{2} v_{i}}{\partial y^{2}}+(n-1) \delta, n \delta\right]$, then $\Gamma^{\gamma, \delta}$ is a supermodular game for any $e \in E^{Q}$.

Since $S_{i} \subset \mathbb{R}^{2}$, it is a sublattice. By Definition 2, payoff function $u_{i}\left(x_{i}, y\right)=$ $v_{i}(y)+\omega_{i}-T_{i}(m, z)$ is $C^{2}$ on $S_{i}$, therefore, the continuity requirement is trivially satisfied.

Since

$$
\frac{\partial^{2} u_{i}}{\partial m_{i} \partial z_{i}}=1, \forall i
$$

by Theorem 1, the payoff function $u_{i}$ is supermodular in $s_{i}$.

To show that $u_{i}$ has increasing differences in $\left(s_{i}, s_{-i}\right)$, we need to check four conditions.

Since $\gamma \geq 1-\min _{i \in N} \frac{\partial^{2} v_{i}}{\partial y^{2}}+(n-1) \delta$, we have

$$
\frac{\partial^{2} u_{i}}{\partial m_{i} \partial m_{j}}=\frac{\partial^{2} v_{i}}{\partial y^{2}}+\gamma-1-(n-1) \delta \geq 0, \forall i \neq j .
$$

Similarly, since $\gamma \leq n \delta$, we have

$$
\frac{\partial^{2} u_{i}}{\partial m_{i} \partial z_{j}}=-\frac{\gamma}{n}+\delta \geq 0, \forall i \neq j
$$


The two conditions, $\gamma \geq 1-\min _{i \in N} \frac{\partial^{2} v_{i}}{\partial y^{2}}+(n-1) \delta$ and $\gamma \leq n \delta$ can be satisfied simultaneously as long as $\delta \in\left[1-\min _{i \in N} \frac{\partial^{2} v_{i}}{\partial y^{2}},+\infty\right)$ holds.

The last two conditions are straight forward to check:

$$
\begin{gathered}
\frac{\partial^{2} u_{i}}{\partial z_{i} \partial m_{j}}=1, \forall i \neq j ; \text { and } \\
\frac{\partial^{2} u_{i}}{\partial z_{i} \partial z_{j}}=0, \forall i \neq j .
\end{gathered}
$$

By Theorem 1, the payoff function $u_{i}$ has increasing differences. Therefore, $\Gamma^{\gamma, \delta}$ is a supermodular game for any $e \in E^{Q}$.

(ii) Next, we prove that if $\Gamma^{\gamma, \delta}$ is a supermodular game for any $e \in E^{Q}$, then $\delta \in\left[1-\min _{i \in N} \frac{\partial^{2} v_{i}}{\partial y^{2}},+\infty\right)$ and $\gamma \in\left[1-\min _{i \in N} \frac{\partial^{2} v_{i}}{\partial y^{2}}+(n-1) \delta, n \delta\right]$.

If $\Gamma^{\gamma, \delta}$ is a supermodular game for any $e \in E^{Q}$, then $u_{i}$ has increasing differences in $\left(s_{i}, s_{-i}\right)$. Since $u_{i}$ is $C^{2}$, by Theorem $1, u_{i}$ has increasing differences in $\left(s_{i}, s_{-i}\right)$ if and only if all of the following four inequalities hold,

$$
\frac{\partial^{2} u_{i}}{\partial m_{i} \partial m_{j}} \geq 0, \quad \frac{\partial^{2} u_{i}}{\partial m_{i} \partial z_{j}} \geq 0, \quad \frac{\partial^{2} u_{i}}{\partial z_{i} \partial m_{j}} \geq 0, \text { and } \frac{\partial^{2} u_{i}}{\partial z_{i} \partial z_{j}} \geq 0, \quad \forall i \neq j .
$$

From part (i), we know that the first two inequalities imply that

$$
n \delta \geq \gamma \geq 1-\min _{i \in N} \frac{\partial^{2} v_{i}}{\partial y^{2}}+(n-1) \delta
$$

which in turn implies that $\delta \in\left[1-\min _{i \in N} \frac{\partial^{2} v_{i}}{\partial y^{2}},+\infty\right)$.

Q.E.D.

Since the new mechanism is supermodular with a proper choice of parameters under a class of quasilinear preferences, it has a robust dynamic stability property. Therefore, compared with the Groves-Ledyard mechanism, it has the advantage of implementing Lindahl allocations. Compared with other mechanisms implementing the Lindahl allocations, it has a robust stability property. Compared with the Abreu-Matsushima mechanism which is dominance-solvable but with a huge message space, the new mechanism is simple with only a twodimensional message space. Note in this entire class of Nash-efficient public goods mechanisms only the Groves-Ledyard mechanism and the Walker mechanism are balanced both on and off the equilibrium path. All others are balanced only in equilibrium.

Under the new mechanism as well as the Groves-Ledyard mechanism, when choosing parameters to induce supermodularity, the planner needs to know the smallest second partial derivative of the players' utility for public goods in the society, i.e., $\min _{i \in N} \frac{\partial^{2} v_{i}}{\partial y^{2}}$, for all possible levels of the public good, $y$. Note that by Definition 1 this term is bounded below. Note also that this is state-dependent information. In Nash implementation theory we usually assume that the planner does not have any information about the players' preferences. In that case, even though there exist a set of stable mechanisms among a family of mechanisms, 
the planner does not have the information to choose the right one. Therefore, in order to choose parameters to implement the stable set of mechanisms, the planner needs to have some information about the distribution of preferences and an estimate about the possible range of public goods level.

\section{Concluding remarks}

So far Nash implementation theory has mainly focused on establishing static properties of the equilibria. However, experimental evidence suggests that the fundamental question concerning any actual implementation of a specific mechanism is whether decentralized dynamic learning processes will actually converge to one of the equilibria promised by theory. Based on its attractive theoretical properties $^{8}$ and the supporting evidence for these properties in the experimental literature, we focus on supermodularity as a robust stability criterion for Nashefficient public goods mechanisms.

We present a new family of Nash mechanisms which implement Lindahl allocations in a general environment; with quasilinear utility functions the new family of mechanisms are supermodular games given a suitable choice of parameters. Thus theoretically the new mechanisms have similar stability properties as the Groves-Ledyard mechanism and are also individually rational.

Two aspects of the convergence and stability analysis in this paper warrant attention. First, supermodularity is sufficient but not necessary for convergence to hold. It is possible that a mechanism could fail supermodularity but still behaves well on a class of adjustment dynamics, such as the Kim mechanism. Secondly, The stability analysis in this paper, like other theoretical studies of the dynamic stability of Nash mechanisms, have been restricted to quasilinear utility functions. It is desirable to extend the analysis to other more general environments. The maximal domain of stable environments remains an open question.

\section{Appendix}

To prove Theorem 2, we need Lemmas 1 to 5 .

Lemma 1 If $(\bar{m}, \bar{z})$ is a Nash equilibrium of $\Gamma^{\gamma, \delta}$ for $e \in E$, then $\bar{z}_{i}=\sum_{k=1}^{n} \bar{m}_{k}$ and $T_{i}(\bar{m}, \bar{z})=P_{i}(\bar{m}, \bar{z}) \cdot Y(\bar{m}, \bar{z})$, for all $i$.

Proof. Since $(\bar{m}, \bar{z})$ is a Nash equilibrium, then for each $i$,

$$
\left[\omega_{i}^{x}-T_{i}(\bar{m}, \bar{z}), Y(\bar{m}, \bar{z})\right] \succeq_{i}\left[\omega_{i}^{x}-T_{i}\left(\bar{m}, \bar{z}_{-i}, z_{i}\right), Y\left(\bar{m}, \bar{z}_{-i}, z_{i}\right)\right]
$$

for all $z_{i}$. Since preferences are strictly increasing in private good $x$, for each $i$,

\footnotetext{
${ }^{8}$ In particular, Milgrom and Roberts (1990) have shown that a supermodular game converges under a wide class of learning dynamics, including Bayesian learning, fictitious play, adaptive learning, Cournot best response and many others.
} 


$$
\bar{z}_{i}=\sum_{k=1}^{n} \bar{m}_{k} .
$$

It follows that

$$
T_{i}(\bar{m}, \bar{z})=P_{i}(\bar{m}, \bar{z}) \cdot Y(\bar{m}, \bar{z}) .
$$

Q.E.D.

Lemma 2 Continuity, local nonsatiation and convexity of preferences, and continuity of $T_{i}(m, z)$ in $\left(m_{i}, z_{i}\right)$ imply that $X_{i}(\bar{m}, \bar{z})+T_{i}(\bar{m}, \bar{z})=\omega_{i}^{x}$ for all $i$, where $(\bar{m}, \bar{z})$ is a Nash equilibrium of $\Gamma^{\gamma, \delta}$ for $e \in E$.

Proof. See Groves and Ledyard (1977) p.799.

Lemma 3 Let $A_{i}=\left\{\left(X_{i}, Y\right) \in \mathscr{C}_{i} \mid\left(X_{i}, Y\right) \succeq_{i}\left(\bar{X}_{i}, \bar{Y}\right)\right\}$. If $\succeq_{i}$ are complete, transitive and convex, then $A_{i}$ is convex.

Proof. Take $\left(X_{i}, Y\right) \in \mathscr{C}_{i},\left(\bar{X}_{i}, \bar{Y}\right) \in \mathscr{C}_{i}$ and $\left(X_{i}^{\prime}, Y^{\prime}\right) \in \mathscr{C}_{i}$. Suppose $\left(X_{i}, Y\right) \succeq_{i}$ $\left(\bar{X}_{i}, \bar{Y}\right)$ and $\left(X_{i}^{\prime}, Y^{\prime}\right) \succeq_{i}\left(\bar{X}_{i}, \bar{Y}\right)$. Let $\hat{X}_{i}=\alpha X_{i}+(1-\alpha) X_{i}^{\prime}, \hat{Y}=\alpha Y+(1-\alpha) Y^{\prime}$, where $\alpha \in[0,1]$. Then $\left(\hat{X}_{i}, \hat{Y}\right) \in \mathscr{C}_{i}$ since $\mathscr{C}_{i}$ is a convex set. We next want to show that $\left(\hat{X}_{i}, \hat{Y}\right) \succeq_{i}\left(\bar{X}_{i}, \bar{Y}\right)$. Since preferences are complete, without loss of generality, we can assume $\left(X_{i}, Y\right) \succeq_{i}\left(X_{i}^{\prime}, Y^{\prime}\right)$. By convexity of preferences, we have $\left(\hat{X}_{i}, \hat{Y}\right) \succeq_{i}\left(X_{i}^{\prime}, Y^{\prime}\right)$, which by transitivity yields $\left(\hat{X}_{i}, \hat{Y}\right) \succeq_{i}\left(\bar{X}_{i}, \bar{Y}\right)$. Q.E.D.

To prove the Pareto optimality of the Nash equilibrium, we need a minimum wealth result, i.e., no player is in her minimum wealth condition in equilibrium. In equilibrium although any strictly preferred point must be outside the budget set, since the budget set is strictly convex along the boundary the separating hyperplane may contain strictly preferred points. This possibility is ruled out by Lemma 4.

Lemma 4 Under Assumption 2, if $(\bar{m}, \bar{z})$ is a Nash equilibrium of the mechanism $\Gamma^{\gamma, \delta}$, for each $i$ there exists $\left(m_{i}, z_{i}\right)$ such that $\left[X_{i}\left(\bar{m}_{-i}, \bar{z}_{-i}, m_{i}, z_{i}\right)\right.$, $\left.Y\left(\bar{m}_{-i}, \bar{z}_{-i}, m_{i}, z_{i}\right)\right] \in \mathscr{C}_{i}$ and $X_{i}\left(\bar{m}_{-i}, \bar{z}_{-i}, m_{i}, z_{i}\right)+T_{i}\left(\bar{m}_{-i}, \bar{z}_{-i}, m_{i}, z_{i}\right)<X_{i}(\bar{m}, \bar{z})+$ $T_{i}(\bar{m}, \bar{z})$.

Proof. Let $\bar{X}_{i}=X_{i}(\bar{m}, \bar{z})$ and $\bar{Y}=Y(\bar{m}, \bar{z})$. Assumption 2 implies that $\bar{X}_{i}>0$ and $\bar{Y}>0$. Let $m_{i}=\bar{m}_{i}$ and $z_{i}=\bar{Y}+\sqrt{2 \epsilon}$ for some $\epsilon>0$ such that $\bar{X}_{i}-\epsilon>0$. Since $(\bar{m}, \bar{z})$ is a Nash equilibrium, we have $T_{i}(\bar{m}, \bar{z})=P_{i}(\bar{m}, \bar{z}) \cdot Y(\bar{m}, \bar{z})$ by Lemma 1 , and

$$
T_{i}\left(\bar{m}_{-i}, \bar{z}_{-i}, m_{i}, z_{i}\right)=T_{i}(\bar{m}, \bar{z})+\frac{1}{2}\left(z_{i}-\bar{Y}\right)^{2}=T_{i}(\bar{m}, \bar{z})+\epsilon .
$$

Let

$$
\hat{X}_{i}=\omega_{i}-T_{i}\left(\bar{m}_{-i}, \bar{z}_{-i}, m_{i}, z_{i}\right)=\omega_{i}-T_{i}(\bar{m}, \bar{z})-\epsilon=\bar{X}_{i}-\epsilon>0 .
$$

Therefore, there exists $\sigma>0$ such that

$$
X_{i}\left(\bar{m}_{-i}, \bar{z}_{-i}, m_{i}, z_{i}\right)=\hat{X}_{i}-\sigma>0 .
$$


By construction $Y\left(\bar{m}_{-i}, \bar{z}_{-i}, m_{i}, z_{i}\right)=\bar{Y}>0$, therefore, $\left[X_{i}\left(\bar{m}_{-i}, \bar{z}_{-i}, m_{i}, z_{i}\right)\right.$, $\left.Y\left(\bar{m}_{-i}, \bar{z}_{-i}, m_{i}, z_{i}\right)\right] \in \mathscr{C}_{i}$. Eq. (1) and (2) imply that

$$
X_{i}\left(\bar{m}_{-i}, \bar{z}_{-i}, m_{i}, z_{i}\right)+T_{i}\left(\bar{m}_{-i}, \bar{z}_{-i}, m_{i}, z_{i}\right)=\omega_{i}-\sigma<\omega_{i}=X_{i}(\bar{m}, \bar{z})+T_{i}(\bar{m}, \bar{z}) .
$$

Q.E.D.

Lemma 5 If $(\bar{m}, \bar{z})$ is a Nash equilibrium of $\Gamma^{\gamma, \delta}$ for $e \in E$, then $\left[\left(\omega_{i}^{x}-\right.\right.$ $\left.\left.T_{i}(\bar{m}, \bar{z})\right)_{i=1}^{n}, Y(\bar{m}, \bar{z})\right]$ is a Pareto optimal allocation for $e \in E$.

Proof. ${ }^{9}$ : Each player must make three decisions: she must choose a private good consumption bundle $X_{i} \in \mathbb{R}^{1}$, and a pair of messages $\left(m_{i}, z_{i}\right)$ to send to the planner. Nash behavior implies that given others' decisions a player will choose a decision triple $\left(X_{i}, m_{i}, z_{i}\right)$ to maximize preferences over consumption bundle $\left(X_{i}, Y\right)$ subject to a budget constraint.

We define the budget correspondence of player $i$ by

$$
\begin{aligned}
\beta_{i}\left(m_{-i}, z_{-i}\right)= & \left\{\left(X_{i}^{*}, m_{i}^{*}, z_{i}^{*}\right) \in \mathbb{R}^{1} \times S_{i} \mid\left(X_{i}^{*}, Y\left(m_{-i}, z_{-i}, m_{i}^{*}, z_{i}^{*}\right)\right)\right. \\
& \left.\in \mathscr{C}_{i}, X_{i}^{*}+T_{i}\left(m_{-i}, z_{-i}, m_{i}^{*}, z_{i}^{*}\right) \leq \omega_{i}^{x}\right\} .
\end{aligned}
$$

The decision correspondence of player $i$ is defined by

$$
\begin{aligned}
\eta_{i}\left(m_{-i}, z_{-i}\right)= & \left\{\left(X_{i}^{*}, m_{i}^{*}, z_{i}^{*}\right) \in \beta_{i}\left(m_{-i}, z_{-i}\right) \mid\left(X_{i}^{*}, Y\left(m_{-i}, z_{-i}, m_{i}^{*}, z_{i}^{*}\right)\right)\right. \\
& \succeq_{i}\left(X_{i}, Y(m, z)\right)
\end{aligned}
$$$$
\text { for all } \left.\left(X_{i}, m_{i}, z_{i}\right) \in \beta_{i}\left(m_{-i}, z_{-i}\right)\right\} \text {. }
$$

We now prove Lemma 5 in seven steps. Let $\bar{Y} \equiv Y(\bar{m}, \bar{z})$.

1. . $\bar{X}_{i}+T_{i}(\bar{m}, \bar{z})=\omega_{i}^{x}$ for all $i$ (Lemma 2).

2. For any $\left(X_{i}, Y\right) \in \mathscr{C}_{i}$, there is a pair $\left(m_{i}, z_{i}\right)$ such that $Y=Y\left(\bar{m}_{-i}, \bar{z}_{-i}, m_{i}, z_{i}\right)$. Simply let $m_{i}=Y-\sum_{j \neq i} \bar{m}_{j}$.

3. $\left(X_{i}, Y\right) \succeq_{i}\left(\bar{X}_{i}, \bar{Y}\right), Y=m_{i}+\sum_{j \neq i} \bar{m}_{j}$ implies $X_{i}+T_{i}\left(\bar{m}_{-i}, \bar{z}_{-i}, m_{i}, z_{i}\right) \geq$ $\bar{X}_{i}+T_{i}(\bar{m}, \bar{z})$.

If not, $X_{i}+T_{i}\left(\bar{m}_{-i}, \bar{z}_{-i}, m_{i}, z_{i}\right)<\omega_{i}^{x}$ (by 1). Continuity, local nonsatiation, convexity of preferences and continuity of $T_{i}(m, z)$ in $(m, z)$ imply that there is a triple $\left(X_{i}^{\prime}, m_{i}^{\prime}, z_{i}^{\prime}\right)$ such that $\left(X_{i}^{\prime}, Y\left(\bar{m}_{-i}, \bar{z}_{-i}, m_{i}^{\prime}, z_{i}^{\prime}\right)\right) \in$ $\mathscr{C}_{i}, X_{i}^{\prime}+T_{i}\left(\bar{m}_{-i}, \bar{z}_{-i}, m_{i}^{\prime}, z_{i}^{\prime}\right) \leq \omega_{i}^{x}$ and $\left(X_{i}^{\prime}, Y\left(\bar{m}_{-i}, \bar{z}_{-i}, m_{i}^{\prime}, z_{i}^{\prime}\right)\right) \succ_{i}\left(\bar{X}_{i}, \bar{Y}\right)$. Then $\left(\bar{X}_{i}, \bar{m}_{i}, \bar{z}_{i}\right) \notin \eta_{i}\left(m_{-i}, z_{-i}\right)$, which is a contradiction.

4. $\left(X_{i}, Y\right) \succ_{i}\left(\bar{X}_{i}, \bar{Y}\right), Y=m_{i}+\sum_{j \neq i} \bar{m}_{j}$ implies $X_{i}+T_{i}\left(\bar{m}_{-i}, \bar{z}_{-i}, m_{i}, z_{i}\right)>$ $\bar{X}_{i}+T_{i}(\bar{m}, \bar{z})$.

If not, $\left(\bar{X}_{i}, \bar{m}_{i}, \bar{z}_{i}\right) \notin \eta_{i}\left(m_{-i}, z_{-i}\right)$, which is a contradiction.

5. $\left(X_{i}, Y\right) \succeq_{i}\left(\bar{X}_{i}, \bar{Y}\right)$ implies $X_{i}+t_{i} \cdot Y \geq \bar{X}_{i}+t_{i} \cdot \bar{Y}$.

Let $\widehat{T}_{i}\left(Y ; m_{-i}, z\right)=P_{i}\left(m_{-i}, z\right) \cdot Y+\frac{1}{2}\left(Y-z_{i}\right)^{2}+\frac{\delta}{2} \sum_{j \neq i}\left(Y-z_{j}\right)^{2}$. Define $t_{i}=\partial \widehat{T}_{i}\left(Y ; m_{-i}, z\right) / \partial Y=P_{i}+\left(Y-z_{i}\right)+\delta \sum_{j \neq i}\left(Y-z_{j}\right)$. Let $A_{i}=\left\{\left(X_{i}, Y\right) \in\right.$ $\left.\mathscr{C}_{i} \mid\left(X_{i}, Y\right) \succeq_{i}\left(\bar{X}_{i}, \bar{Y}\right)\right\}$. By Lemma $3, A_{i}$ is convex. And $\left(\bar{X}_{i}, \bar{Y}\right)$ is in the

\footnotetext{
${ }^{9}$ The proof is similar to Groves and Ledyard's (1977, p. 799).
} 
boundary of $A_{i}$. Let $B_{i}=\left\{\left(X_{i}, Y\right) \in \mathscr{C}_{i} \mid X_{i}+\widehat{T}_{i}\left(Y ; m_{-i}, z\right) \leq \omega_{i}^{x}\right\}$. Since $\widehat{T}_{i}(\cdot)$ is a convex function of $Y, B_{i}$ is convex. By $1,\left(\bar{X}_{i}, \bar{Y}\right)$ is in the boundary of $B_{i}$. By $4, A_{i}^{\circ} \cap B_{i}^{\circ}=\phi$, that is, the intersection of the interior of set $A_{i}$ and $B_{i}$ is empty. Thus there exists a hyperplane through $\left(\bar{X}_{i}, \bar{Y}\right)$ separating $A_{i}$ and $B_{i}$, and the vector $\left(1, t_{i}\right)$ defines the hyperplane. It follows that $\left(X_{i}, Y\right) \succeq_{i}\left(\bar{X}_{i}, \bar{Y}\right)$ implies $X_{i}+t_{i} \cdot Y \geq \bar{X}_{i}+t_{i} \cdot \bar{Y}$.

6. $\left(X_{i}, Y\right) \succ_{i}\left(\bar{X}_{i}, \bar{Y}\right)$ implies $X_{i}+t_{i} \cdot Y>\bar{X}_{i}+t_{i} \cdot \bar{Y}$.

Suppose not. By $5, X_{i}+t_{i} \cdot Y=\bar{X}_{i}+t_{i} \cdot \bar{Y}$. By 2, 5 and Lemma 4, there exists $(\hat{X}, \hat{Y}) \in \mathscr{C}_{i}$ such that $\hat{X}_{i}+t_{i} \cdot \hat{Y}<\bar{X}_{i}+t_{i} \cdot \bar{Y}$. Let $G=\left\{\left(X_{i}^{\prime}, Y^{\prime}\right) \in\right.$ $\mathscr{C}_{i} \mid\left(X_{i}^{\prime}, Y^{\prime}\right)=\left(\lambda \hat{X}_{i}+(1-\lambda) X_{i}, \lambda \hat{Y}+(1-\lambda) Y\right)$ for all $\left.\lambda \in[0,1]\right\}$. So all points along the line between $\left(\hat{X}_{i}, \hat{Y}\right)$ and $\left(X_{i}, Y\right)$ have lower value than $\left(\bar{X}_{i}, \bar{Y}\right)$. By continuity of preferences there is a neighborhood $N$ of $\left(X_{i}, Y\right)$ such that $\left(X_{i}^{\prime}, Y^{\prime}\right) \in N \cap \mathscr{C}_{i}$ implies that $\left(X_{i}^{\prime}, Y^{\prime}\right) \succ_{i}\left(\bar{X}_{i}, \bar{Y}\right)$. This corresponds to a point in the closure of $N$ with smaller value than $\left(\bar{X}_{i}, \bar{Y}\right)$. But $N \cap G \neq \phi$. This leads to a contradiction of 5 .

7. Suppose $\left[\left(\bar{X}_{i}\right)_{i=1}^{n}, \bar{Y}\right]$ is not Pareto-optimal. Let $\left[\left(X_{i}\right)_{i=1}^{n}, Y\right]$ be a Paretosuperior feasible allocation. It follows from 5 and 6 that $\sum_{i} X_{i}+\sum_{i} t_{i}$. $Y>\sum_{i} \bar{X}_{i}+\sum_{i} t_{i} \cdot \bar{Y}$. In particular, we can set $z_{i}=Y$ for all $i$. Then $\sum_{i} t_{i}=\sum_{i} P_{i}=b$. It follows that

$$
\sum_{i} X_{i}+b \cdot Y>\sum_{i} \bar{X}_{i}+b \cdot \bar{Y}=\sum_{i} \omega_{i}^{x}
$$

Therefore, $\left[\left(X_{i}\right)_{i=1}^{n}, Y\right]$ is not feasible.

Q.E.D.

Proof of Theorem 2. (i) We first show that if $(\bar{m}, \bar{z})$ is a Nash equilibrium of $\Gamma^{\gamma, \delta}$ for $e \in E$, then $\left[\left(P_{i}(\bar{m}, \bar{z}), \omega_{i}^{x}-T_{i}(\bar{m}, \bar{z})\right)_{i=1}^{n}, Y(\bar{m}, \bar{z})\right]$ is a Lindahl equilibrium for $e$ with $P_{i}(\bar{m}, \bar{z})$ as the Lindahl price of the public good for player $i$. Since Lemma 5 show that Nash equilibrium is Pareto-optimal, we only need to show that Pareto optima are Lindahl equilibria. The following argument is similar to that in Foley (1970) p.68. Let $\bar{X}_{i} \equiv \omega_{i}^{x}-T_{i}(\bar{m}, \bar{z})$, and $\bar{Y} \equiv Y(\bar{m}, \bar{z})$. Define

$$
\begin{aligned}
F & =\left\{\left(X_{1}, X_{2}, \cdots, X_{n}, Y_{1}, Y_{2}, \cdots, Y_{n}\right) \mid\left(X_{i}, Y_{i}\right) \in \mathscr{C}_{i}, Y_{i}\right. \\
& \left.=Y_{j}=Y \text { for } i \neq j \text { and } Y \leq \frac{\sum_{i=1}^{n}\left(\omega_{i}^{x}-X_{i}\right)}{b}\right\}
\end{aligned}
$$

It is easy to see that $F$ is a convex set and the point $\left(\bar{X}_{1}, \bar{X}_{2}, \cdots, \bar{X}_{n}, \bar{Y}_{1}, \bar{Y}_{2}, \cdots, \bar{Y}_{n}\right)$ is in the boundary of $F$. Define

$$
D=\left\{\left(X_{1}, X_{2}, \cdots, X_{n}, Y_{1}, Y_{2}, \cdots, Y_{n}\right) \mid\left(X_{i}, Y_{i}\right) \in \mathscr{C}_{i},\left(X_{i}, Y_{i}\right) \succeq_{i}\left(\bar{X}_{i}, \bar{Y}\right), \forall i\right\} .
$$

$D$ is convex via the same argument as Lemma 3 , and the point $\left(\bar{X}_{1}, \bar{X}_{2}, \cdots\right.$, $\left.\bar{X}_{n}, \bar{Y}_{1}, \bar{Y}_{2}, \cdots, \bar{Y}_{n}\right)$ is in the boundary of $D$. The intersection of the interior of the two sets is empty, $F^{\circ} \cap D^{\circ}=\phi$, since if it was not $\left(\left\{\bar{X}_{i}\right\}_{i=1}^{n}, \bar{Y}\right)$ would not be a Pareto optimum, which contradicts Lemma 5. By the separating hyperplane 
theorem, there exists a price vector, $\left(p_{1}^{x}, \cdots, p_{n}^{x}, p_{1}^{y}, \cdots, p_{n}^{y}\right) \neq 0$ and a scalar $c$ such that for all $\left(X_{1}, X_{2}, \cdots, X_{n}, Y_{1}, Y_{2}, \cdots, Y_{n}\right) \in D$,

$$
\sum_{i=1}^{n} p_{i}^{x} \cdot X_{i}+\left(\sum_{i=1}^{n} p_{i}^{y}\right) \cdot Y \geq c
$$

Since $\left(\bar{X}_{1}, \bar{X}_{2}, \cdots, \bar{X}_{n}, \bar{Y}_{1}, \bar{Y}_{2}, \cdots, \bar{Y}_{n}\right) \in D \cap F$, we have

$$
\sum_{i=1}^{n} p_{i}^{x} \cdot \bar{X}_{i}+\left(\sum_{i=1}^{n} p_{i}^{y}\right) \cdot \bar{Y}=c .
$$

By 5 of the proof of Lemma 5 , the hyperplane going through $\left(\bar{X}_{i}, \bar{Y}\right)$ is defined by the vector $\left(1, t_{i}\right)$ for all $i$ where

$$
t_{i}=P_{i}(m, z)+\left(Y-z_{i}\right)+\delta \sum_{j \neq i}\left(Y-z_{j}\right) .
$$

Therefore, $p_{i}^{x}=1$ and $p_{i}^{y}=t_{i}$ for all $i$. Under the assumption of Nash behavior, we have

$$
\left.p_{i}^{y}=P_{i}(\bar{m}, \bar{z})+\left(\bar{Y}-\bar{z}_{i}\right)+\delta \sum_{j \neq i}\left(\bar{Y}-\bar{z}_{j}\right)=P_{i}(\bar{m}, \bar{z}), \text { (by Lemma } 1\right) .
$$

Next we want to show that $\left(\bar{X}_{i}, \bar{Y}\right)$ maximizes the preference of player $i$ subject to the budget constraint. Suppose $\left(X_{i}, Y_{i}\right) \succ_{i}\left(\bar{X}_{i}, \bar{Y}\right)$ while $X_{j}=\bar{X}_{j}$ and $Y_{j}=\bar{Y}$ for all $j \neq i$, then the point $\left(X_{1}, X_{2}, \cdots, X_{n}, Y_{1}, Y_{2}, \cdots, Y_{n}\right)$ is in $D$. From Eq. (3) and (4) we have

$$
\sum_{k=1}^{n} X_{k}+\left(\sum_{k=1}^{n} P_{k}(\bar{m}, \bar{z})\right) \cdot Y \geq \sum_{k=1}^{n} \bar{X}_{k}+\left(\sum_{k=1}^{n} P_{k}(\bar{m}, \bar{z})\right) \cdot \bar{Y} .
$$

Since all terms are the same on both sides except those corresponding to $i$, it follows that $X_{i}+P_{i}(\bar{m}, \bar{z}) \cdot Y \geq \bar{X}_{i}+P_{i}(\bar{m}, \bar{z}) \cdot \bar{Y}$. By the same argument as in 6 of the proof of Lemma 5 , equality cannot hold. Therefore, we have

$$
X_{i}+P_{i}(\bar{m}, \bar{z}) \cdot Y>\bar{X}_{i}+P_{i}(\bar{m}, \bar{z}) \cdot \bar{Y} .
$$

Since $P_{i}(\bar{m}, \bar{z})=P_{i}\left(\bar{m}_{-i}, \bar{z}_{-i}, m_{i}, z_{i}\right)$, Eq. (5) implies

$$
X_{i}+P_{i}\left(\bar{m}_{-i}, \bar{z}_{-i}, m_{i}, z_{i}\right) \cdot Y+\frac{1}{2}\left[Y-z_{i}\right]^{2}+\frac{\delta}{2} \sum_{j \neq i}\left[Y-\bar{z}_{j}\right]^{2}>\bar{X}_{i}+P_{i}(\bar{m}, \bar{z}) \cdot \bar{Y} .
$$

By Lemma 1, this is equivalent to $X_{i}+T_{i}\left(\bar{m}_{-i}, \bar{z}_{-i}, m_{i}, z_{i}\right)>\bar{X}_{i}+T_{i}(\bar{m}, \bar{z})$.

Therefore, $\left(X_{i}, Y\right) \succ_{i}\left(\bar{X}_{i}, \bar{Y}\right)$ implies $X_{i}+T_{i}\left(\bar{m}_{-i}, \bar{z}_{-i}, m_{i}, z_{i}\right)>\bar{X}_{i}+\bar{T}_{i}(\bar{m}, \bar{z})$, i.e., $\left[\omega_{i}^{x}-T_{i}(\bar{m}, \bar{z}), Y(\bar{m}, \bar{z})\right]$ maximizes the preference of player $i$ subject to the budget constraint.

From Lemma 1, it follows that 


$$
\sum_{i=1}^{n} T_{i}(\bar{m}, \bar{z})=b \cdot Y(\bar{m}, \bar{z}) .
$$

Therefore, the allocation $\left[\left(\omega_{i}^{x}-T_{i}(\bar{m}, \bar{z})\right)_{i=1}^{n}, Y(\bar{m}, \bar{z})\right]$ is feasible. Hence, $\left[\left(P_{i}(\bar{m}, \bar{z}), \omega_{i}^{x}-T_{i}(\bar{m}, \bar{z})\right)_{i=1}^{n}, Y(\bar{m}, \bar{z})\right]$ is a Lindahl equilibrium.

(ii) In the second part, we prove that if $\left[\left(\bar{P}_{i}, \bar{X}_{i}\right)_{i=1}^{n}, \bar{Y}\right]$ is a Lindahl equilibrium for $e \in E$, then there is a Nash equilibrium $(\bar{m}, \bar{z})$ of $\Gamma^{\gamma, \delta}$ for $e$, such that $Y(\bar{m}, \bar{z})=\bar{Y}, \omega_{i}^{x}-T_{i}(\bar{m}, \bar{z})=\bar{X}_{i}$, and $\bar{P}_{i}(\bar{m}, \bar{z})=\bar{P}_{i}$.

Let $\left[\left(\bar{P}_{i}, \bar{X}_{i}\right)_{i=1}^{n}, \bar{Y}\right]$ be a Lindahl equilibrium. Let $\bar{z}_{i}=\bar{Y}$ for all $i$ and $\left(\bar{m}_{i}\right)_{i=1}^{n}$ be the solution to the following $n$ linear equations:

$$
\left\{\begin{aligned}
\bar{m}_{1}+\cdots+\bar{m}_{n} & =\bar{Y} \\
-\gamma \sum_{j \neq i} \bar{m}_{j} & =\bar{P}_{i}-\frac{b}{n}-\frac{\gamma}{n} \sum_{j \neq i} \bar{z}_{j}, \text { for } i=1, \ldots, n-1 .
\end{aligned}\right.
$$

Note that the above system has a unique solution $\left(\bar{m}_{i}\right)_{i=1}^{n}$. Since $\left[\left(\bar{P}_{i}, \bar{X}_{i}\right)_{i=1}^{n}, \bar{Y}\right]$ is a Lindahl equilibrium,

$$
\left[\omega_{i}^{x}-\bar{P}_{i} \cdot \bar{Y}, \bar{Y}\right] \succeq_{i}\left[\omega_{i}^{x}-\bar{P}_{i} \cdot Y, Y\right] \text {, for all } Y .
$$

Choose $Y=m_{i}+\sum_{j \neq i} \bar{m}_{j}$. Then for each $i$,

$$
\left[\omega_{i}^{x}-\bar{P}_{i} \cdot \bar{Y}, \bar{Y}\right] \succeq_{i}\left[\omega_{i}^{x}-\bar{P}_{i} \cdot\left(m_{i}+\sum_{j \neq i} \bar{m}_{j}\right), m_{i}+\sum_{j \neq i} \bar{m}_{j}\right], \text { for all } m_{i} .
$$

Since preferences are strictly increasing in the private good $x$, we have

$$
\begin{gathered}
{\left[\omega_{i}^{x}-\bar{P}_{i} \cdot\left(m_{i}+\sum_{j \neq i} \bar{m}_{j}\right), m_{i}+\sum_{j \neq i} \bar{m}_{j}\right] \succeq_{i}\left[\omega_{i}^{x}-\bar{P}_{i} \cdot\left(m_{i}+\sum_{j \neq i} \bar{m}_{j}\right)-\frac{1}{2}\left(z_{i}-m_{i}-\sum_{j \neq i} \bar{m}_{j}\right)^{2}\right.} \\
\left.-\frac{\delta}{2} \sum_{j \neq i}\left(\bar{z}_{j}-m_{i}-\sum_{j \neq i} \bar{m}_{j}\right)^{2}, m_{i}+\sum_{j \neq i} \bar{m}_{j}\right]
\end{gathered}
$$

for all $m_{i}, z_{i}$. From Eq. (7) and (8), it follows that

$$
\begin{gathered}
{\left[\omega_{i}^{x}-\bar{P}_{i} \cdot \bar{Y}, \bar{Y}\right] \succeq_{i}\left[\omega_{i}^{x}-\bar{P}_{i} \cdot\left(m_{i}+\sum_{j \neq i} \bar{m}_{j}\right)-\frac{1}{2}\left(z_{i}-m_{i}-\sum_{j \neq i} \bar{m}_{j}\right)^{2}\right.} \\
\left.-\frac{\delta}{2} \sum_{j \neq i}\left(\bar{z}_{j}-m_{i}-\sum_{j \neq i} \bar{m}_{j}\right)^{2}, m_{i}+\sum_{j \neq i} \bar{m}_{j}\right],
\end{gathered}
$$

for all $m_{i}, z_{i}$. From Eq. (6), for all $i$,

$$
\begin{gathered}
\bar{Y}=\sum_{k=1}^{n} \bar{m}_{k}=Y(\bar{m}, \bar{z}), \\
\bar{P}_{i}=\frac{b}{n}-\gamma \sum_{j \neq i} \bar{m}_{j}+\frac{\gamma}{n} \sum_{j \neq i} \bar{z}_{j}=P_{i}(\bar{m}, \bar{z})=P_{i}\left(\bar{m}_{-i}, \bar{z}_{-i}, m_{i}, z_{i}\right),
\end{gathered}
$$

Therefore, from Eq. (9) and (10), we have 


$$
\begin{aligned}
& {\left[\omega_{i}^{x}-P_{i}(\bar{m}, \bar{z}) \cdot Y(\bar{m}, \bar{z})-\frac{1}{2}\left(\bar{z}_{i}-\sum_{k=1}^{n} \bar{m}_{k}\right)^{2}\right.} \\
& \left.\quad-\frac{\delta}{2} \sum_{j \neq i}\left(\bar{z}_{j}-\sum_{k=1}^{n} \bar{m}_{k}\right)^{2}, Y(\bar{m}, \bar{z})\right] \succeq_{i} \\
& {\left[\omega_{i}^{x}-P_{i}\left(\bar{m}_{-i}, \bar{z}_{-i}, m_{i}, z_{i}\right) \cdot Y\left(\bar{m}_{-i}, \bar{z}_{-i}, m_{i}, z_{i}\right)-\frac{1}{2}\left(z_{i}-m_{i}-\sum_{j \neq i} \bar{m}_{j}\right)^{2}\right.} \\
& \left.\quad-\frac{\delta}{2} \sum_{j \neq i}\left(\bar{z}_{j}-m_{i}-\sum_{j \neq i} \bar{m}_{j}\right)^{2}, Y\left(\bar{m}_{-i}, \bar{z}_{-i}, m_{i}, z_{i}\right)\right]
\end{aligned}
$$

for all $m_{i}, z_{i}$. Hence $(\bar{m}, \bar{z})$ is a Nash equilibrium of $\Gamma^{\gamma, \delta}$, with $Y(\bar{m}, \bar{z})=\bar{Y}$, $\omega_{i}^{x}-T_{i}(\bar{m}, \bar{z})=\bar{X}_{i}$, and $P_{i}(\bar{m}, \bar{z})=\bar{P}_{i}$.

Q.E.D.

Proof of Corollary 1. (i) We first show that if $(\bar{m}, \bar{z})$ is a Nash equilibrium of $\Gamma^{\gamma, \delta}$ for $e \in E^{Q}$, then $\left[\left(P_{i}(\bar{m}, \bar{z}), \omega_{i}^{x}-T_{i}(\bar{m}, \bar{z})\right)_{i=1}^{n}, Y(\bar{m}, \bar{z})\right]$ is a Lindahl equilibrium for $e$ with $P_{i}(\bar{m}, \bar{z})$ as the Lindahl price of the public good for player $i$.

Maximizing the utility function, holding $\bar{m}_{-i}$ and $\bar{z}_{-i}$ fixed,

$$
\max _{m_{i}, z_{i}} v_{i}(Y)+\omega_{i}^{x}-T_{i}\left(\bar{m}_{-i}, \bar{z}_{-i}, m_{i}, z_{i}\right)
$$

yields a first order condition

$$
z_{i}=m_{i}+\sum_{j \neq i} \bar{m}_{j}
$$

Using Eq. (11) and the Envelope Theorem we obtain the other first order condition,

$$
D v_{i}(Y)-P_{i}\left(\bar{m}_{-i}, \bar{z}_{-i}, m_{i}, z_{i}\right)+\delta \sum_{j \neq i}\left(\bar{z}_{j}-m_{i}-\sum_{k \neq i} \bar{m}_{k}\right)=0 .
$$

In Nash equilibrium, $m_{i}=\bar{m}_{i}$ and $z_{i}=\bar{z}_{i}$, then Eq. (12) yields

$$
D v_{i}(\bar{Y})=P_{i}(\bar{m}, \bar{z}) \text {. }
$$

Feasibility is verified in the proof of Theorem 2. Therefore, $\left[\left(P_{i}(\bar{m}, \bar{z}), \omega_{i}^{x}-\right.\right.$ $\left.\left.T_{i}(\bar{m}, \bar{z})\right)_{i=1}^{n}, Y(\bar{m}, \bar{z})\right]$ is a Lindahl equilibrium for $e \in E^{Q}$ with $P_{i}(\bar{m}, \bar{z})$ as the Lindahl price of the public good for player $i$.

(ii) If $\left[\left(\bar{P}_{i}, \bar{X}_{i}\right)_{i=1}^{n}, \bar{Y}\right]$ is a Lindahl equilibrium for $e \in E^{Q}$, then there is a Nash equilibrium $(\bar{m}, \bar{z})$ of $\Gamma^{\gamma, \delta}$ for $e$, such that $Y(\bar{m}, \bar{z})=\bar{Y}, \omega_{i}^{x}-T_{i}(\bar{m}, \bar{z})=\bar{X}_{i}$, and $\bar{P}_{i}(\bar{m}, \bar{z})=\bar{P}_{i}$. This part is identical to part (ii) of the proof of Theorem 2, since it does not require Assumption 2.

Q.E.D.

Proof of Corollary 2. Summing Eq. (13) over $i$ yields

$$
\sum_{i} D v_{i}(\bar{Y})=b
$$


Since, by Definition $2, D^{2} v_{i}(Y)<0$ for all $i$, there can be at most one value of $\bar{Y}$ that satisfies Eq. (14). Let this value be $Y^{*}=\sum_{i} m_{i}^{*}$.

Definition 2, $D v_{i}(Y)>0$ for all $i$, and Eq. (13) imply that $P_{i}^{*}$ is also unique. By Lemma $1, z_{i}^{*}=\sum_{i} m_{i}^{*}=Y^{*}$, then $z_{i}^{*}$ is unique. Eq. (10) can be rearranged as

$$
m_{i}^{*}=\frac{P_{i}^{*}}{\gamma}+\frac{Y^{*}}{n}-\frac{b}{\gamma n}
$$

which solves uniquely for $m_{i}^{*}$.

Q.E.D.

\section{References}

Abreu, D., Matsushima, H.: Virtual implementation in iteratively undominated strategies: complete information. Econometrica 60(3), 993-1008 (1992)

Arrow, K., Hurwicz, L.: Stability of the Gradient Process in N Person Games. In: Arrow, K., Hurwicz, L. (eds.) Studies in resource allocation processes. Cambridge: Cambridge University Press 1977

Bagnoli, M., Lipman, B.: Provision of public goods: fully implementing the core through private contributions. Review of Economic Studies 56, 583-602 (1989)

Boylan, R., El-Gamal, M.: Fictitious play: a statistical study of multiple economic experiments. Games and Economic Behavior 5, 205-222 (1993)

Cabrales, A.: Adaptive dynamics and the implementation problem with complete information. Journal of Economic Theory 86, 159-184 (1999)

Chen, Y.: Dynamic stability of Nash-efficient public goods mechanisms: reconciling theory with experiments. Games and Economic Behavior (forthcoming)

Chen, Y.: Incentive-compatible mechanisms for pure public goods: a survey of experimental research. In: Plott, C., Smith, V. (eds) The handbook of experimental economics results. Amsterdam: Elsevier (forthcoming)

El-Gamal, M., Grether, D.: Uncovering behavioral strategies: are people Bayesians? Journal of the American Statistical Associations 90, 1137-1145 (1995)

Falkinger, J.: Efficient private provision of public goods by rewarding deviations from average. Journal of Public Economics 62, 413-422 (1996)

Green, J., Laffont, J.-J.: Characterization of satisfactory mechanisms for the revelation of the preferences for public goods. Econometrica 45, 427-438 (1977)

Groves, T., Ledyard, J.: (1977) Optimal allocation of public goods: a solution to the 'free rider' problem. Econometrica 45(4), 783-809 (1977)

Hurwicz, L.: On informationally decentralized systems. In: McGuire, C., Radner, R. (eds.) Decision and organization, pp. 297-336. Amsterdam: North Holland 1972

Hurwicz, L.: Outcome functions yielding Walrasian and Lindahl allocations at Nash equilibrium points. Review of Economic Studies 46, 217-225 (1979)

Jackson, M., Moulin, H.: Implementing a public project and distributing its costs. Journal of Economic Theory 57, 125-140 (1992)

Kim, T.: On the nonexistence of a stable Nash mechanism implementing Lindahl allocations. University of Minnesota, mimeo (1986)

Kim, T.: A stable Nash mechanism implementing Lindahl allocations for quasi-linear environments. Journal of Mathematical Economics 22, 359-371 (1993)

Ledyard, J.: Public goods: a survey of experimental research. In: Kagel, J., Roth, A. (eds) Handbook of experimental economics. Princeton, NJ: Princeton University Press 1995

Milgrom, P., Roberts, J.: Rationalizability, learning and equilibrium in games with strategic complementarities. Econometrica 58(6), 1255-1277 (1990)

Milgrom, P., Roberts, J.: Adaptive and sophisticated learning in normal form games. Games and Economic Behavior 3, 82-100 (1991)

Muench, T., Walker, M.: (1983) Are Groves-Ledyard equilibria attainable? Review of Economic Studies L, 393-396 (1983) 
Peleg, B.: Double implementation of the Lindahl equilibrium by a continuous mechanism. Economic Design 2, 311-324 (1996)

Roberts, J.: Incentives and planning procedures for the provision of public goods. Review of Economic Studies 46, 283-292 (1979)

Tian, G.: Implementation of the Lindahl correspondence by a single-valued, feasible, and continuous mechanism. Review of Economic Studies 56, 613-621 (1989)

Topkis, D.: Minimizing a submodular function on a lattice. Operations Research 26, 305-321 (1978)

de Trenqualye, P.: Stability of the Groves-Ledyard mechanism. Journal of Economic Theory 46, 164-171 (1988)

de Trenqualye, P.: Stable implementation of Lindahl allocations. Economic Letters 29, 291-294 (1989)

Van Huyck, J., Battalio, R., Beil, R.: Tacit coordination games, strategic uncertainty, and coordination failure. American Economic Review 80, 234-248 (1990)

Vega-Redondo, F.: Implementation of Lindahl equilibrium: an integration of static and dynamic approaches. Mathematical Social Sciences 18, 211-228 (1989)

Walker, M.: On the impossibility of a dominant strategy mechanism to optimally decide public questions. Econometrica 48, 1521-1540 (1980)

Walker, M.: A simple incentive compatible scheme for attaining Lindahl allocations. Econometrica 49, 65-71 (1981) 